\title{
The Design of Mass Customization Oriented Laboratory Simulation Platform in IGRIP
}

\author{
Wenqian Zhang ${ }^{1,2}$, Jiawei Xiong ${ }^{1}$, Siyu Chen ${ }^{1}$ and Shuhai Fan ${ }^{1,2, *}$ \\ ${ }^{1}$ Industrial Engineering Department, Nanjing Tech University, Nanjing 210009, China \\ ${ }^{2}$ MIT Quality Information Program "Data Quality \& Info Security"Lab, Cambridge, MA, USA \\ ${ }^{*}$ Corresponding author
}

\begin{abstract}
In order to solve the problem of lack of online simulation and remote control in the mass customization oriented laboratory simulation platform, it is designed by taking IGRIP software as an example. Based on IGRIP, three kinds of interface methods were compared and analyzed for the exchange of information between IGRIP and external systems. The laboratory simulation platform was secondary development. And it was applied in the case of the mass production process for the implementation of the specific function, which fully embodied the advantage of IGRIP in simulation, improving the scope of application effectively, simplifying the procedure of operation to enhance the convenience and operability of the application process for laboratory simulation platform. During the process of simulation design and operation, operators can make full use of what they have learned to solve practical problems, improve research efficiency and stimulate interest in learning.
\end{abstract}

Keywords-mass customization; laboratory simulation platform; online simulation; remote control; IGRIP

\section{INTRODUCTION}

Mass customization (MC) is a production mode which provides customized products and services in a mass production with low cost, high quality and efficiency according to the individual requirements of customers. At present, the manufacturing industry is faced with a difficult problem that is how to meet the needs of various of consumer demands for mass customization to obtain corporate profits. With the continuous development of modern science and technology, the improvement of industrial automation and mechanization, using simulation technology has become the main research technique of industrial production to simulate devices and units[1].

With the help of simulation technology in mass customization, the advance design and operation test can not only reduce the cost of simulation, but also strengthen the ability of data collection and processing, improve the feasibility of the scheme and reduce the enterprise risk[2-3]. In the meantime, the development of virtual simulation technology has also been paid attention by many fields such as science, education and so on.

The mass customization oriented laboratory simulation platform has many mature ergonomic simulation software such as CATIA, JACK, IGRIP and other professional software[4-5]. They have capabilities of graphical simulation and offline programming, but do not directly provide capabilities of online simulation and remote control, so that they need to use their accompanying open programming interfaces and simulation environment customization interface to make the secondary development. In this way, the application scope of software can be extended, and the convenience and operability of the simulation application process can be improved. This paper aims at the design of the mass customization oriented laboratory simulation platform in IGRIP.

\section{SYSTEM ANALYSIS}

Interactive Graphics Robot Instruction Program (IGRIP) is a large commercial digital manufacturing software, which uses internal model libraries to model and establish kinematic relationships quickly, and to perform kinematic simulation and analysis of dynamic processes. The dynamic simulation process in IGRIP consists of two types: discrete and continuous, which are simultaneous. The discrete process is controlled by the GSL language and implemented the GSL instruction through the relevant device. But the continuous process is realized by the function of the device in conjunction with the model's parameters and calling the functions in the dynamic link library written in the relevant $C$ language.

Graphic Simulation Language (GSL) is a programming language used in graphic simulations. GSL is a structured, Pascal-like procedural language. Like Pascal, the program is written using many of the same terms that would be used to state the solution to the original problem. GSL is used to program the actions and behavior of individual devices in a simulation. It is embedded in the system to control the behavior of one or a group of robot devices in the work units, and has its own data type, structure and syntax.

Command Line Interpreter (CLI) is a command language that interacts with some simulation platform. It is a "control" language in the sense that it can behave as a "user" of the system outside to perform a simulation. It can load a work unit, manipulate devices, paths, parts, and run a simulation, etc. This is in contrast to GSL, which is usually focused on the motion of a particular robot device or a group of robot devices within a simulation.

From an overall point of view, the instructions sent by the IGRIP system are composed of discrete points. However, when each discrete point instruction is executed, its execution is continuous. In the implementation of a discrete point instruction into the continuous process control, the first step is setting up the initial parameters based on the current state of the model. Then inverse motion planning is solved according to the 
target value to simulate. The next step is returning calling point to prepare to execute the next discrete point and start the new continuous control process. The simulating program flowchart is shown in figure 1:

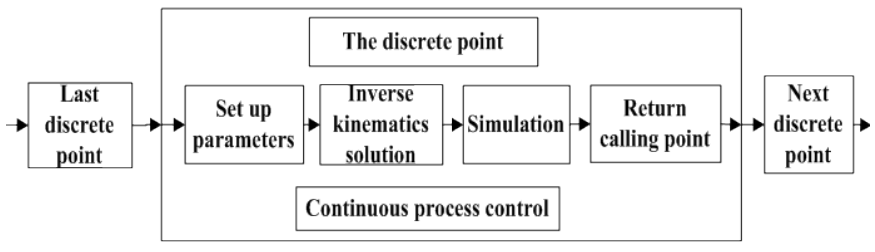

FIGURE I. SIMULATING PROGRAM FLOWCHART

\section{SYSTEM DESIGN}

The system design of IGRIP laboratory simulation platform is divided into three modules as shown in figure 2 :

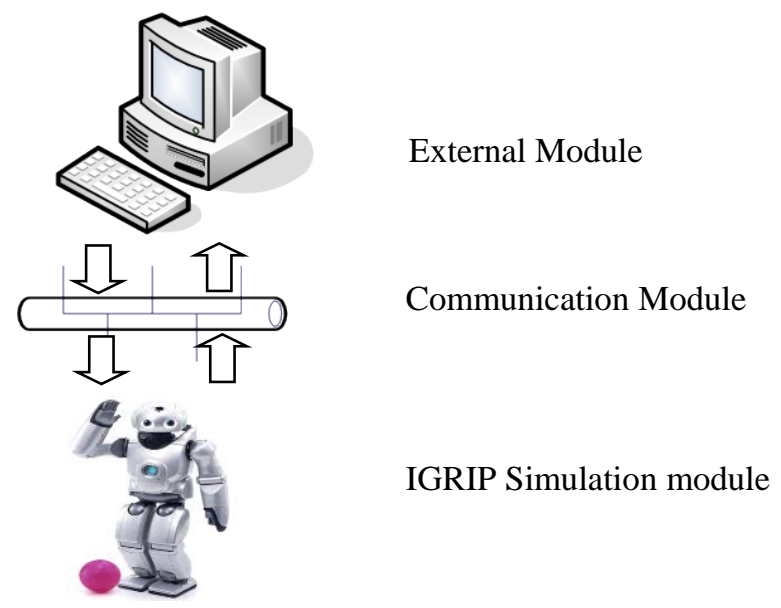

FIGURE II. SYSTEM DESIGN OF IGRIP PLATFORM

In the picture above, the bottom layer is the IGRIP system simulation module. The intermediate layer is the communication module between the simulation software platform and the external programs, and the top layer is the external module[6]. The design of laboratory simulation platform focuses on the the middle layer module that is the communication between the IGRIP system module and the external module. In addition, IGRIP system communicates with external programs in 3 different ways and interfaces, as shown in table 1:
TABLE I. THREE METHODS OF IGRIP COMMUNICATION

\begin{tabular}{|c|c|c|}
\hline & Method & Details \\
\hline \multirow{4}{*}{ GSL } & File & Open a BINARY or TEXT file \\
\hline & Socket & $\begin{array}{l}\text { Initiate a two-way communication link } \\
\text { to establish communication }\end{array}$ \\
\hline & Pipe & Bond UNIX to data exchange \\
\hline & $\begin{array}{l}\text { Dynamic Link } \\
\text { Library(DLL) }\end{array}$ & $\begin{array}{l}\text { Call the } C \text { language function written } \\
\text { by users in the shared library }\end{array}$ \\
\hline CLI & $\begin{array}{c}\text { Low Level } \\
\text { Telerobotics } \\
\text { Interface(LLTI) }\end{array}$ & $\begin{array}{l}\text { Online parallel monitoring technology } \\
\text { to remote control of external } \\
\text { equipment for real-time control and } \\
\text { two-way communication }\end{array}$ \\
\hline & Motion Pipe & $\begin{array}{l}\text { Define the motion components for the } \\
\text { respective modules }\end{array}$ \\
\hline
\end{tabular}

\section{Detailed Design}

According to the three implementations that IGRIP laboratory simulation platform accesses external programs, the IGRIP system is detailed designed in this paper based on the windows system, which is designed from two different ways of interactive: offline and online.

\section{A. Interactive Offline System of Design}

The openness of IGRIP determines the choice of file method, so it can be simulated directly by opening the file. It allows operators to open a BINARY or TEXT file for three types of operations:input, output, and append. Input specifies that the file is to be opened for reading only, and output and append specify that the file is to be opened for writing. For example, the specific instructions for opening the file "File" are as follows:

PROGRAM File

VAR file: STRING

Main Declaration Section

\section{BEGIN MAIN}

OPEN FILE 'D:IDeneb\Syslib\PROGRAMS Iworker \Model.gsl’ FOR TEXT INPUT AS 1

while ( READ_LINE( \#1, file) $<>$ \$EOF ) do

write(file, $\mathrm{cr}$ )

endwhile

--------- END MAIN ----------
END File

\section{B. Interactive Online System of Design}

Operators and the IGRIP platform communicate commands between the client and the server to perform online interaction by creating two-way communications via sockets, enabling operators to understand the various real-time status during the 
simulation. In the client with TCP/IP protocol, the web is used as input interface of the client, and operators login robot simulation home page and enter the CLI commands to transfer it to the IGRIP computer to ensure the instantaneity and interactiveness of the simulation robot and achieve the purpose of online transmitting the data information quickly and accurately. Specific instructions are as follows:

\section{1) Create a socket}

\section{OPEN SOCKET 'robot1:D:|Deneb\EXAMPLES \ PROGRAMS \rob_socket’ FOR UPDATE AS 2}

\section{OPEN CLIENT ‘user:2017’ FOR UPDATE AS 2 \\ OPEN SERVER 2017 FOR UPDATE AS 2 \\ CLOSE \#2}

This statement is used to initiate a two-way communication link on either the current host computer or a remote host connected via TCP/IP. Moreover, communication may be established either by executing a command on a given host or by binding a TCP port address to a server process. The flow of socket communication is shown in figure 3 :

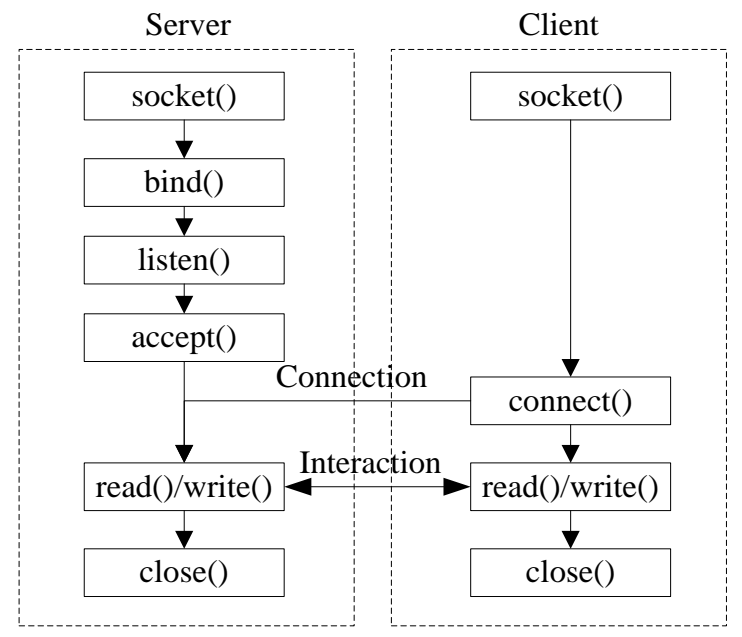

FIGURE III. THE FLOW CHART OF SOCKET COMMUNICATION

\section{2) Initialization routine of simulation}

Igrip-s<2017>

retrieve workcell "IW"

IW= 'robot1'

load program “Model.gsl” into IW

activate IW

The simulation robot model can be controlled on-line through the above steps.

\section{APPLIED ANALYSIS}

Taking an example of simulation about manual materials handing in a mass production process of an automobile mechanical manufacturing company, the simulation is carried out on the IGRIP laboratory platform in combination with the above methods. The simulation diagram is built as shown in figure 4:

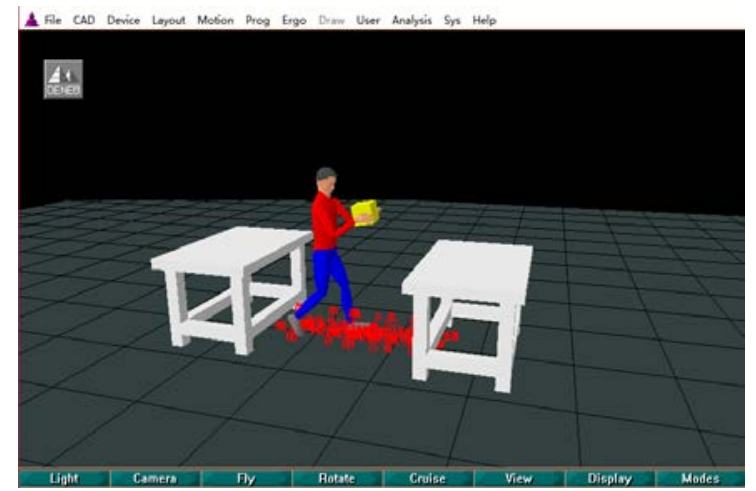

FIGURE IV. THE MODEL OF SIMULATION

In the simulation, the worker carries the needed materials from the table to the working table by manual handling in the loading and unloading of the body welding process, then return to the end point. First of all, it needs to be built with the worker's model, work environment, workflow, a series of specific poses and so on during manual materials handling. The worker moves from the start point to the table, then grabs the material and lifts it to the working table, places it at the working table, finally returns to the end point where the process ends. The instruction input and feedback windows are shown in figures 5 and 6.

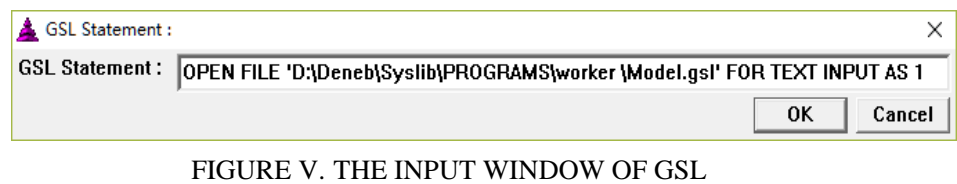

\begin{tabular}{|l|l|}
\hline A. filestart_window & - \\
\hline The model will work
\end{tabular}

FIGURE VI. THE FEEDBACK WINDOW OF GSL

Its GSL program is as follows:

PROGRAM Model

VAR path1,path2: PATH

Main Declaration Section

\#INCLUDE anthro

\section{BEGIN MAIN}

open window 'model_window' @ 0.5, 0.5: 8 as 1

write@1,( ‘the model will work', cr )

UNITS = INCH

\$SPEED_MODE $=$ PERCENT

\$SPEED $=1$

MOVE ALONG 'path1' 
WRITE('It took' ,\$cycle_time, 'seconds to move along path1',cr)

\$CYCLE_TIME=0

MOVE ALONG 'path2'

END MAIN

\section{END Model}

The results of simulation about the worker's energy consumption are summarized as shown in table 2. By comparison, the result can be drawn that the worker's average energy consumption rate is highest in the third phase when he lifts the material, whose value is $4.131 \mathrm{Kcal} / \mathrm{min}[7]$. In addition, the second is the forth phase whose value is $3.692 \mathrm{Kcal} / \mathrm{min}$.

On the basis of simulation results, the larger phases of energy consumption can be improved to optimize the overall production process from the point of relevant professional knowledge.

TABLE II. THE RESULTS OF ENERGY CONSUMPTION

\begin{tabular}{|c|c|c|}
\hline Task & Details & $\begin{array}{c}\text { Average energy } \\
\text { consumption/Kcal/ } \\
\text { min }\end{array}$ \\
\hline 1 & Start & 2.385 \\
\hline 2 & Lift arms & 2.635 \\
\hline 3 & Lift materials & 4.131 \\
\hline 4 & Send materials to the working table & 3.692 \\
\hline 5 & Lower arms & 2.625 \\
\hline 6 & Place materials on the working table & 3.065 \\
\hline 7 & Return & 2.377 \\
\hline
\end{tabular}

VI. SUMMARY

Mass customization oriented Laboratory simulation platform allows operators to obtain direct perceptual knowledge in the process of simulation design and operation to further understand the professional knowledge and consolidate the knowledge learned in school. In addition, operators can make full use of what they have learned to solve practical problems, improve research efficiency and stimulate interest in learning.

Based on the platform of IGRIP laboratory simulation software, this paper compares and analyzes three different kinds of supportive interfaces for information exchange between it and external systems. It is designed from two different ways: offline and online to make it efficient for information interaction. Moreover, the specific case is carried out in the mass production process of an automobile manufacturing company to embody it give full play to the advantages of simulation on the laboratory platform. These methods can effectively expand the scope of application, simplify the simulation process, and enhance the convenience and operability of the application process for laboratory simulation platforms.

\section{ACKNOWLEDGMENT}

This work was supported by National Natural Science Foundation of China (Grant No.71671089,71171110,71371097) and Postgraduate Practical Innovation Program in JiangSu(SJCX17_0300).

\section{REFERENCES}

[1] Huang Yin-di, Bian Rong-hua, Zhang Jun. "Study on Logistics Simulation Software Application at Home and Abroad," Industrial Engineering and Management, Jun.2010,Vol.15,No.3,pp.124-128.

[2] Fan Shu-hai, Xiao Tian-yuan, Qiao Gui-xiu. "A DENEB-QUEST Based Quality Simulation System.” Journal of System Simulation,May 2002, Vol.14, No.5,pp:607-608.

[3] Shikdar, Ashraf, S. Al-Araimi, and B. Omurtag. "Development of a software package for ergonomic assessment of manufacturing industry." Pergamon Press, Inc. 2002.

[4] Hunter, Steve L. "Ergonomic evaluation of manufacturing system designs.” Journal of Manufacturing Systems, 2002,Vol.20.No.6,pp:429444.

[5] Jiang Min, Shan Jia-fang, Kong Jun. "Rearch of the communication based on socket between multiple platforms." Computer Engineering and Applications, 2005,Vol.41,No.36,pp:135-137.

[6] FAN Shu-hai, Xiao Tian-yuan, Qiao Gui-xiu, “A DENEB-QUEST Based Quality Simulation System,” Journal of System Simulation, May 2002,Vol.14,No.5,pp.607-608+616.

[7] Garg, A, D. B. Chaffin, and G. D. Herrin.,"Prediction of metabolic rates for manual materials handling jobs,” Aihaj. 1978,39.8,pp.661-674. 